\title{
Icariin Protects against Glucocorticoid- Induced Osteonecrosis of the Femoral Head in Rats
}

\author{
Zengfa Huang $^{\mathrm{a}}$ Cheng Cheng $^{\mathrm{b}}$ Beibei Caoc Jing Wang ${ }^{\mathrm{b}}$ Hui Wei ${ }^{\mathrm{b}}$ \\ Xianzhe Liu ${ }^{\mathrm{b}}$ Yu Han ${ }^{\mathrm{b}}$ Shuhua Yang ${ }^{\mathrm{b}}$ Xiang Wanga \\ a'Department of Radiology, The Central Hospital of Wuhan, Tongji Medical College, Huazhong \\ University of Science and Technology, Wuhan, 'bepartment of Orthopedics, Union Hospital, Tongji \\ Medical College, Huazhong University of Science and Technology, Wuhan, 'Department of Community \\ Health, Hanyang District Center for Disease Control and Prevention, Wuhan, China
}

\section{Key Words}

Glucocorticoid (GC) • Osteonecrosis of the femoral head (ONFH) • Icariin (ICA) • Runx2 • PPARY

\begin{abstract}
Background/Aims: Glucocorticoid (GC)-related osteonecrosis of the femoral head (ONFH) is a common complication following administration of steroids to treat many diseases. Our previous study demonstrated that icariin (ICA) might have a beneficial effect on the bone marrow mesenchymal stem cells (BMSCs) of patients with steroid-associated osteonecrosis. In this study, we investigated the underlying mechanisms of ICA associated with the potential enhancement of osteogenesis and anti-adipogenesis in GC-related ONFH. Methods: In vitro cell proliferation was evaluated by CCK-8 assay. Alizarin red S and alkaline phosphatase (ALP) activity were used to measure osteogenic differentiation, while adipogenic differentiation was revealed by oil red $O$ staining and TG content assay. The expression level of osteogenesis-associated genes and PPARY was evaluated by RT-qPCR, western blotting and immunofluorescence. A total of 30 female SD rats were randomly separated into three groups: a control group, a methylprednisolone (MPS) group and a MPS + ICA group. Serum ALP and TG (triglyceride), micro-CT scanning, histological and immunohistochemical analyses were performed in the animal model. Results: In the in vitro study, ICA promoted proliferation, improved osteogenic differentiation and suppressed adipogenic differentiation of BMSCs treated with MPS. The group treated with MPS and $10^{-6} \mathrm{M}$ ICA expressed higher levels of Runx2, ALP, bone morphogenetic protein (BMP) 2, and OC and lower expression of PPARY than the MPS group. In the in vivo study, ICA prevented bone loss in a rat model of GC-related ONFH as shown by micro-CT scanning, histological and immunohistochemical analyses. Conclusions: ICA is an effective compound for promoting bone repair and preventing or delaying the progression of GC-associated ONFH in rats. This effect can be explained by its ability to improve the balance between adipogenesis and osteogenesis, indicating that ICA is an effective candidate for management of GC-associated ONFH.




\section{Introduction}

Glucocorticoid (GC)-related osteonecrosis of the femoral head (ONFH) is a common complication following administration of steroids in many diseases, including systemic lupus erythematosus, rheumatoid arthritis, multiple myeloma, acute lymphoblastic leukemia, organ transplantation and severe acute respiratory syndrome [1-6]. There are many mechanisms of GC-related ONFH such as disruption of the balance between osteogenic and adipogenic differentiation [7], fat embolization [8], circulatory impairment [9], cell apoptosis and dysfunction [10], intramedullary pressure changes [11], coagulation disorders [12], and modified artery constriction [13]. Among them, disruption of the balance between osteogenic and adipogenic differentiation of bone marrow mesenchymal stem cells (BMSCs) is a principal mechanism involved in the onset and progression of this disease. When adipocytes increase in number and enlarge, the intraosseous pressure may rise and blood flow rate may decrease, resulting in reduced bone remodeling, causing ONFH. Hence, it is essential for the treatment of GC-related ONFH to restore the balance between osteogenic and adipogenic differentiation of BMSCs, by inhibiting adipogenesis and promoting osteogenesis and thus increasing bone remodeling [14].

Previous epidemiological data showed a remarkable difference in the prevalence of osteonecrosis (mainly knee and hip) in patients recovering from SARS between southern China (5-6\%) [15, 16] and northern China (32.7\%) [17]. The lower prevalence of osteonecrosis in southern China was suggested to be associated with the potential use of conventional herbal intervention to prevent GC-associated osteonecrosis. The antiviral Herba Epimedii with immunomodulation is one of the medicinal herbs which has been widely used as a "Bone Strengthener and Kidney Tonic" in China for thousands of years [18, 19]. Icariin (ICA) is extracted from Herba Epimedii and is considered to be the major active ingredient. It has been reported that ICA can improve bone mineral density (BMD) and bone strength in ovariectomized rats [20]. Moreover, Li et al. reported that ICA prevented ovariectomyinduced bone loss and lowered marrow adipogenesis [21]. Meanwhile our previous study demonstrated that ICA might have a beneficial effect on the BMSCs of patients with steroidassociated osteonecrosis by demethylation of the adenosine triphosphate-binding cassette (ABC) B1-promoter [14]. However, to date, few investigations have focused on the relevance of ICA to the balance between osteogenic and adipogenic differentiation in the treatment of GC-related ONFH.

The aim of this study was to investigate the effect of ICA on potential treatment to prevent progression of ONFH. In this study, we hypothesized that ICA could prevent the development of GC-induced $\mathrm{ONFH}$ by regulating the balance between osteogenic and adipogenic differentiation of BMSCs. Furthermore, we also evaluated the underlying mechanisms of ICA associated with the potential enhancement of osteogenesis and anti-adipogenesis involving BMSCs using methylprednisolone (MPS) to suppress osteogenic activity both in vitro and in vivo.

\section{Materials and Methods}

\section{Cell culture}

Bone marrow mesenchymal stem cells (BMSCs) were obtained from the bilateral femurs and tibias of 3-week-old female Sprague-Dawley (SD) rats (Laboratory Animal Center of Huazhong University of Science and Technology, Wuhan, China) according to the method described by Kodama et al. [22]. The cells were cultured in $\alpha$ minimum essential medium ( $\alpha$-MEM; Gibco BRL, Grand Island, NY, USA) supplemented with $10 \%$ fetal bovine serum (FBS; Invitrogen, Carlsbad, CA, USA) and $1 \%$ penicillin G-streptomycin at $37^{\circ} \mathrm{C}$ in a humidified atmosphere of $5 \% \mathrm{CO}_{2}$. BMSCs were passaged when adherent cells reached a density of 80-90\%. BMSCs at passages three to six were used in all experiments. All procedures involving animals were approved by the Institutional Animal Care and Use Committee of Tongji Medical College, Huazhong University of Science and Technology.

\section{KARGER}




\section{Cellular Physiology Cell Physiol Biochem 2018;47:694-706 \begin{tabular}{l|l|} 
and Biochemistry Published online: May 25, 2018 & $\begin{array}{l}\text { C } 2018 \text { The Author(s). Published by S. Karger AG, Basel } \\
\text { www.karger.com/cpb }\end{array}$ \\
\hline
\end{tabular} \\ Huang et al.: Icariin Protects Against Osteonecrosis}

ICA (purity > 98\%) was purchased from Abcam, Cambridge, UK. It was dissolved in dimethyl sulfoxide (DMSO) and stored in the dark at $-20^{\circ} \mathrm{C}$. The final concentration of DMSO in the medium was $0.1 \%$ or less throughout the experiments. All experiments were repeated three times.

\section{Cell proliferation and viability}

The effect of ICA on cell proliferation was evaluated using the Cell Counting Kit-8 (CCK-8; Nanjing Jiancheng Bioengineering Institute, Nanjing, China) assay according to the manufacturer's instructions. BMSCs were plated into 96-well plates $(5,000$ cells/well $)$ and treated with ICA $\left(10^{-9} \mathrm{M}\right.$ to $\left.10^{-3} \mathrm{M}\right)$ for 48 hours. The results were presented as absorbance read at $450 \mathrm{~nm}$ using a multifunctional microplate reader (ELX800, BioTeK Instruments Inc., Winooski, VT, USA).

To assay cell viability, BMSCs were plated into 96-well plates (five wells per group) at the same cell density and divided into five groups: (1) control group; (2) MPS group, which was treated with $5 \times 10^{-5} \mathrm{M}$ MPS (methylprednisolone, Pfizer, New York, USA); (3) MPS + ICA ${ }^{-5}$ group; treated with $5 \times 10^{-5}$ M MPS and $10^{-5}$ M ICA; (4) MPS + ICA ${ }^{-6}$ group; treated with $5 \times 10^{-5}$ M MPS and $10^{-6}$ M ICA; (5) MPS + ICA ${ }^{-7}$ group; treated with $5 \times 10^{-5} \mathrm{M}$ MPS and $10^{-7} \mathrm{M}$ ICA. The relative cell activity was measured by CCK- 8 assay at 48 and 72 hours. The MPS concentration was chosen based on previous studies [23].

\section{Osteogenic and adipogenic induction}

BMSC differentiation was induced after the adherent cells reached a density of $80-90 \%$. For osteogenic induction, basic medium was supplemented with $10^{-7} \mathrm{M}$ dexamethasone, $10^{-2} \mathrm{M}$ sodium $\beta$-glycerophosphate and $50 \mu \mathrm{g} / \mathrm{mL} \mathrm{L}$-ascorbic acids. For adipogenic induction, BMSCs were cultured in adipogenic medium (culture medium supplemented with dexamethasone $\left(10^{-7} \mathrm{M}\right)$, indomethacin $\left(6 \times 10^{-6} \mathrm{M}\right)$, insulin $(5 \mu \mathrm{g} /$ $\mathrm{mL})$, and 3-isobutyl-1- methylxanthine $\left.\left(5 \times 10^{-4} \mathrm{M}\right)\right)$. The medium was changed every 3 days for 2 weeks to induce BMSC differentiation.

For CCK-8, alkaline phosphatase (ALP) activity and triglyceride (TG) content assays, BMSCs from all five groups were assayed. For alizarin red S staining, oil red 0 staining, immunofluorescence staining, western blotting and RT-qPCR, BMSCs from three groups were assayed: control group, MPS group and MPS + ICA $\left(10^{-6} \mathrm{M}\right)$ group. All experiments were repeated three times, with three replicate wells in each group.

\section{Alizarin red $S$ and oil red $O$ staining}

After osteogenic induction for 14 days, cells in 12-well plates (three replicate wells per group, three plates) were washed with PBS three times and fixed with $4 \%$ paraformaldehyde for 30 min. After fixation, paraformaldehyde was removed from the wells and cells were washed with $\mathrm{ddH}_{2} \mathrm{O}$ three times. Then, 0.04 $\mathrm{M}$ alizarin red $\mathrm{S}$ was used to stain cells for $30 \mathrm{~min}$ at room temperature (RT). After rinsing twice with $\mathrm{ddH}_{2} \mathrm{O}$, cells were visualized under a light microscope (three plates).

After adipogenic induction for 14 days (three replicate wells per group, three plates), cells were gently washed with PBS three times, and fixed with 4\% paraformaldehyde for $30 \mathrm{~min}$. After fixation, paraformaldehyde was removed from the wells and cells were washed with $\mathrm{ddH}_{2} \mathrm{O}$ three times. Then, the cells were stained with oil red 0 staining solution for $30 \mathrm{~min}$ at RT. Thereafter, the samples were photographed under an inverted microscope.

\section{Alkaline phosphatase (ALP) activity and triglyceride (TG) content assay}

BMSCs were seeded into 24-well plates (three replicate wells per group) at a density of 10, 000 cells/ well and cultured with osteogenic or adipogenic medium. ALP activity was assayed on days 3, 7 and 14 at a wavelength of $405 \mathrm{~nm}$ using a multifunctional microplate reader. ALP activity was quantified in cell lysates using an ALP assay kit (Nanjing Jiancheng Biological Engineering Institute) according to the manufacturer's instructions (three plates). TG content was assayed on day 14 using a triglyceride assay kit (Nanjing Jiancheng Biological Engineering Institute) according to the manufacturer's instructions at a wavelength of $510 \mathrm{~nm}$ using a multifunctional microplate reader (three plates).

\section{Immunofluorescence}

BMSCs were seeded onto round cover slips and placed into 12-well plates (three replicate wells per group, three plates). After 2 weeks of osteogenic or adipogenic induction, they were fixed with $4 \%$ paraformaldehyde for $20 \mathrm{~min}$, treated with $0.1 \%$ Triton X-100 for $20 \mathrm{~min}$ and blocked with goat serum 


\section{Cellular Physiology Cell Physiol Biochem 2018:47:694-706

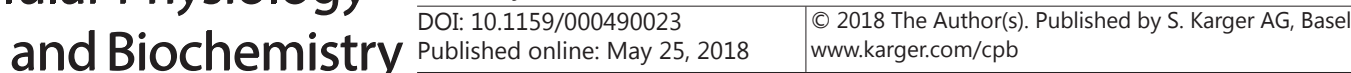

Huang et al.: Icariin Protects Against Osteonecrosis

for $30 \mathrm{~min}$ at $37^{\circ} \mathrm{C}$. Then, cells were washed three times with PBS and incubated with primary antibodies (mouse monoclonal anti-Runx2, anti-PPAR $\gamma$; Abcam) overnight at $4^{\circ} \mathrm{C}$. After rinsing with PBS three times, these

Table 1. Real time PCR gene markers

\begin{tabular}{lll}
\hline Gene Makers & \multicolumn{1}{c}{ Forward $\left(5^{\prime}-3^{\prime}\right)$} & \multicolumn{1}{c}{ Reverse $\left(5^{\prime}-3^{\prime}\right)$} \\
\hline GAPDH & ACCACAGTCCATGCCATCAC & TCCACCCTGTTGCTGTA \\
Runx2 & AGCGGACGAGGCAAGAGTTT & AGGCGGGACACCTACTCTCATA \\
OC & TCAACAATGGACTTGGAGCCC & AGCTCGTCACAATTGGGGTT \\
ALP & CAAGGATGCTGGGAAGTCCG & CTCTGGGCGCATCTCATTGT \\
BMP2 & AGGATGCTGGGAAGTCCG & AGGTGCCACGATCCAGTCAT \\
P-gp & GCCTGGCAGCTGGAAGACAAATAC & ATGGCCAAAATCAAGGGTTAGC \\
PPAR $\gamma$ & CCCTTTACCACGGTTGATTTC & CTTCAATCGGATGGTTCTTCG \\
\hline
\end{tabular}

slips were incubated with an appropriate Alexa Fluor $^{\mathrm{TM}} 488$

horseradish-peroxidase-conjugated antibody (1:1, 000, Invitrogen) for $1 \mathrm{~h}$ at $37^{\circ} \mathrm{C}$. Finally, slips were stained with 4', 6-diamidino-2-phenylindole (DAPI) for another $10 \mathrm{~min}$, rinsed with PBS and analyzed under a fluorescence microscope (DP72, Olympus, Shinjuku, Tokyo, Japan).

Quantitative real-time polymerase chain reaction (RT-qPCR) and western blot analysis

For RT-qPCR, total RNA was extracted with TRIzol (Invitrogen) according to the manufacturer's instructions, and $1 \mu \mathrm{g}$ of total RNA was used for RT reaction with EasyScript one-step gDNA Removal and cDNA Synthesis Supermix (TransGen Biotech, Beijing, China). The mRNA was quantified by real-time RT-qPCR using the SYBR Green/ROX qRT-PCR mix (Takara, Tokyo, Japan) and the Applied Biosystems StepOnePlus ${ }^{\text {TM }}$ Real-Time PCR System. The sequences of the PCR primers for Runx2, Osteocalcin (OC), ALP, bone morphogenetic protein (BMP) 2, permeability glycoprotein (P-gp), peroxisome proliferator-activated receptor gamma (PPAR $\gamma$ ) and the housekeeping gene glyceraldehyde-3-phosphate dehydrogenase (GAPDH) are listed in Table 1. After denaturing DNA templates at $95^{\circ} \mathrm{C}$ for $10 \mathrm{~min}$, the reactions were followed by 40 cycles at $95^{\circ} \mathrm{C}$ for $15 \mathrm{~s}, 60^{\circ} \mathrm{C}$ for $20 \mathrm{~s}$ and $75^{\circ} \mathrm{C}$ for $10 \mathrm{~s}$. Relative expression levels were determined by the $2^{-\Delta \Delta \mathrm{Ct}}$ method.

For western blot analysis, cells were lysed in RIPA buffer (Sigma-Aldrich, St. Louis, MO, USA). Equal quantities of protein were loaded and separated on an SDS-PAGE gel, and transferred onto PVDF membranes. After blocking in TBST containing 5\% fat-free milk for $1 \mathrm{~h}$ at RT, the membranes were probed overnight at $4^{\circ} \mathrm{C}$ with the following primary antibodies: mouse monoclonal anti-GAPDH, anti-Runx2, antiALP, anti-BMP2, anti-OC, anti-P-gp and anti-PPARy; all from Abcam. After being washed, the membranes were incubated with relevant horseradish peroxide-conjugated secondary antibodies $(1: 10,000$, Dako Cytomation, Glostrup, Denmark) for $1 \mathrm{~h}$ at RT. GAPDH was used as loading control. The protein bands were visualized and detected by the ECL system (Pierce, Rockford, IL, USA).

\section{Animal model and grouping}

A total of 30 female SD rats (Laboratory Animal Center of Huazhong University of Science and Technology) were randomly separated into three groups: the MPS group (rats were intravenously injected with $10 \mu \mathrm{g} / \mathrm{kg} / \mathrm{d}$ lipopolysaccharide (LPS, Sigma) for 2 days; after $24 \mathrm{~h}$, the rats were injected intramuscularly with $20 \mathrm{mg} / \mathrm{kg} / \mathrm{d}$ MPS for three consecutive days, $\mathrm{n}=10$ ), the MPS + ICA group (rats were injected with LPS and MPS as in the MPS group accompanied by consecutive ICA $30 \mathrm{mg} / \mathrm{kg} / \mathrm{d}$ feed, $\mathrm{n}=10$ ), the control group (rats were injected with $0.9 \%$ normal saline (equal volume to the MPS group) $n=10$ ). The intervention time was 12 weeks.

At the end of the treatment, blood samples from all the groups were obtained from the tail vein to assay the biochemical parameters. Samples of femoral head were obtained after the rats were sacrificed.

\section{Serum ALP and TG}

Blood samples were centrifuged at 5, $000 \mathrm{rpm}$ for $10 \mathrm{~min}$ at $4^{\circ} \mathrm{C}$. The serum was separated, and the serum ALP activity was measured using a specific assay (Nanjing Jiancheng Biological Engineering Institute) according to the manufacturer's instructions. The absorbance at $405 \mathrm{~nm}$ was recorded as relative ALP levels in rats. Serum concentrations of triglyceride (TG) were measured with a full automatic biochemical analyzer (Roche Modular-T; Roche, Basel, Switzerland).

\section{Micro-CT scanning}

After removing soft tissue, the entire femoral head of each rat was scanned at $15 \mu \mathrm{m}$ isotropic voxel size by micro CT (Scanco Medical, Brüttisellen, Switzerland) to evaluate bone morphologic changes. Images 
were reconstructed in 3D using 3D Creator software (Volume Graphics GmbH, Heidelberg, Germany). The region of interest (ROI) for the analysis and comparison of trabecular parameters was determined according to Dong et al [24]. The trabecular bone parameters including bone mineral density (BMD), bone volume (BV), bone volume/total volume (BV/TV), bone surface/total volume (BS/TV), trabecular separation (Tb. $\mathrm{Sp}$ ), trabecular thickness (Tb.Th) and trabecular number (Tb.N) were quantified to determine the relative amount of bone within the femoral head.

\section{Histological and immunohistochemical analyses}

After micro-CT scanning, the femoral heads were treated by decalcification and paraffin embedding. Then they were sectioned at a thickness of $5 \mu \mathrm{m}$ in the coronal plane. Sections of the left femoral heads in each group were stained with hematoxylin and eosin (H\&E). Oil red 0 was carried out to detect the lipid droplet formation in adipogenesis, the sections of femoral heads were stained with oil red 0 working solution for 15 min after fixing and pre-wetting with $60 \%$ isopropanol. The positive area was quantitative measured with the Image J 1.47 software. The diagnosis of osteonecrosis was established based on the presence of empty lacunae or pyknotic nuclei of osteocytes in the bone trabeculae, accompanied by surrounding bone marrow necrosis [25]. Sections of the right femoral heads were deparaffinized, antigen retrieved, incubated with primary antibodies (mouse monoclonal anti-Runx2, anti-PPAR $\gamma$; Santa Cruz Biotechnology) and then incubated with the appropriate horseradish peroxide-conjugated secondary antibodies. Finally, sections were colored with DAB and counterstained with hematoxylin. Photomicrographs were acquired using a Leica DM 4000. Then, Image-Pro Plus 6.0 was used for quantitative analysis, while target protein and total area of trabecular bones was measured based on integrated optical density (IOD). The results were defined as mean density (IOD/area).

\section{Statistical analysis}

Data were presented as mean $\pm S D$. Differences between groups were analyzed by one-way ANOVA, using SPSS 17.0 software (SPSS, Inc., Chicago, IL, USA). $P<0.05$ was considered as statistically significant.

\section{Results}

\section{ICA promoted BMSC proliferation}

We first examined the individual effects of ICA $\left(10^{-9} \mathrm{M}-10^{-3} \mathrm{M}\right)$ on proliferative activity in BMSCs by CCK8 assay (Fig. 1A). ICA at $10^{-3} \mathrm{M}$ significantly decreased cell proliferation compared to the control group $(P<0.05)$. In contrast, lower concentrations of ICA $\left(10^{-9} \mathrm{M}-10^{-5}\right.$ $\mathrm{M})$ significantly increased cell proliferation $(P<0.05)$. ICA at $10^{-4} \mathrm{M}$ increased cell proliferation, but the difference was not statistically significant $(P>0.05)$. Our results indicated that ICA promoted proliferation of BMSCs, especially at $10^{-6} \mathrm{M}$.

The CCK8 assay showed that BMSC proliferation was significantly suppressed by $5 \times 10^{-5}$ M MPS, while this inhibition was partly antagonized by ICA, especially at a concentration of $10^{-6} \mathrm{M}$ (Fig. 1B).

ICAimproved osteogenic differentiation of BMSCS treated with MPS

ALP activity is a marker of osteoblastic differentiation. As shown in Fig. 2A, after treatment of BMSCs with MPS or MPS

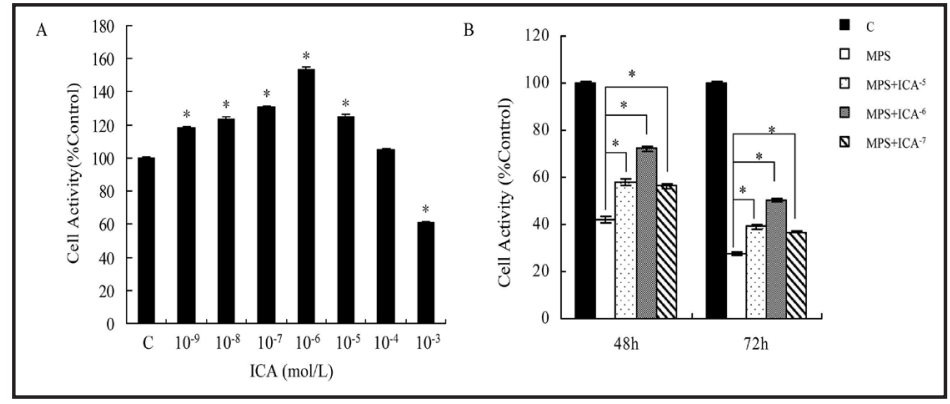

Fig. 1. Effect of ICA, MPS and MPS combined with ICA on cell proliferation activity in BMSCs. (A) After treatment with ICA $\left(10^{-9}-10^{-3}\right.$ M) for $48 \mathrm{~h}$, the proliferation rate of BMSCs was assessed by the CCK-8 assay. Results were obtained from three independent experiments and were expressed as mean $\pm \mathrm{SD}$. ${ }^{*} \mathrm{P}<0.05$ vs. Control group. (B) BMSC proliferation was assayed by CCK- 8 at 48 and $72 \mathrm{~h}$ after incubation with MPS $\left(5 \times 10^{-5} \mathrm{M}\right)$ or MPS $\left(5 \times 10^{-5} \mathrm{M}\right)$ plus different concentrations of ICA. Results were expressed as mean $\pm \mathrm{SD}$. ${ }^{*} \mathrm{P}<0.05$ vs. MPS group. 
+ ICA $\left(10^{-5} \mathrm{M}-10^{-7} \mathrm{M}\right)$ for 3, 7 or 14 days, ALP activity was significantly down-regulated by MPS $(P<0.05)$, while the suppression was antagonized when combined with different concentrations of ICA, especially at $10^{-6} \mathrm{M}(P<0.05)$.

Alizarin red S staining showed fewer calcium nodules in the MPS group compared with the control group, while the mineralization of BMSCs improved significantly in those treated with a combination of ICA and MPS compared to those treated with MPS alone (Fig. 2B).

We analyzed the expression of osteogenic-associated genes by RT-qPCR to verify whether ICA improved osteogenic differentiation of BMSCs treated with MPS. We found that mRNA levels of Runx2, ALP, BMP2 and OC were all significantly down-regulated by MPS $(P<$ $0.05)$, while ICA at $10^{-6} \mathrm{M}$ partly antagonized this down-regulation of Runx2, ALP, BMP2 and OC expression (Fig. 2C). Western blotting showed that MPS down-regulated the expression of Runx2, ALP, BMP2 and OC, while MPS and $10^{-6}$ M ICA increased their expression (Fig. 2D). Immunofluorescence staining revealed that the MPS group exhibited a decrease in Runx2 expression compared to the control group, while the MPS $+\mathrm{ICA}^{-6}$ M group showed an increase in the protein compared to the MPS group (Fig. 4A).

Fig. 2. Effects of MPS and MPS + ICA on osteogenic differentiation of BMSCs. (A) Alkaline phosphatase (ALP) activity was measured on days 3,7 and 14 of incubation in osteogenic medium with MPS or MPS + ICA, and results were shown as mean $\pm \mathrm{SD}$. ${ }^{*} \mathrm{P}<0.05$ vs. MPS group. (B) Alizarin red S staining of BMSCs treated with MPS or MPS + ICA. (C) The mRNA expression of Runx2, OC, ALP and BMP2 in BMSCs were assayed by RT-qPCR after 2 weeks of incubation in osteogenic medium with MPS or

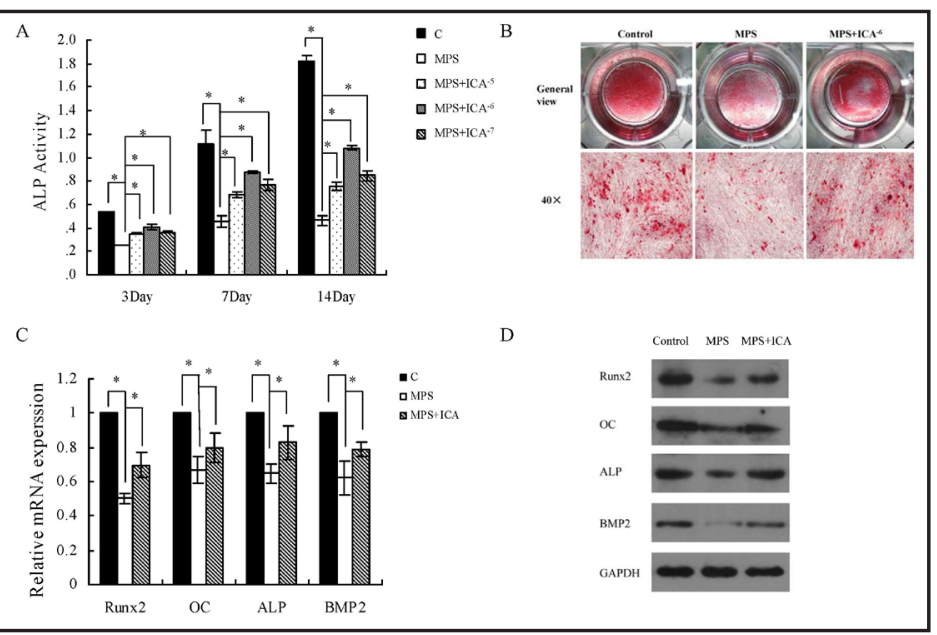
MPS + ICA; results were shown as mean $\pm \mathrm{SD}$. $* \mathrm{P}<0.05$ vs. MPS group. (D) Western blotting results of Runx2, OC, ALP and BMP2 gene expression after 2 weeks of incubation in osteogenic medium with MPS or MPS + ICA.

Fig. 3. Effects of MPS or MPS + ICA on adipogenic differentiation of BMSCs. (A) TG content was measured on day 14 of incubation in adipogenic medium with MPS or MPS + ICA, results were shown as mean \pm SD. * $\mathrm{P}<0.05$ vs. MPS group. (B) Oil red 0 staining of BMSCs treated with MPS or MPS + ICA. (C) The mRNA expressions of P-gp and PPAR $\gamma$ of BMSCs was assayed by RT-qPCR after 2 weeks of incubation in adipogenic medium with MPS or MPS + ICA; results were shown as mean $\pm \mathrm{SD}$. $^{*} \mathrm{P}<0.05$ vs. MPS group. (D) Western blotting results of $\mathrm{P}$-gp and PPAR $\gamma$ gene expression after 2 weeks of incubation in adipogenic medium with MPS or MPS + ICA.

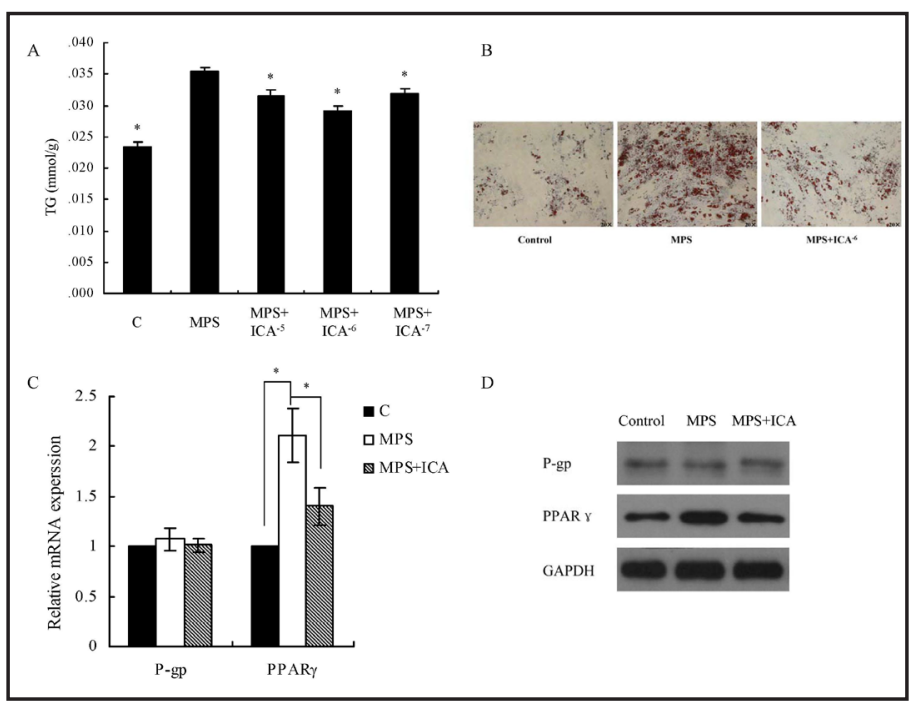




\section{Cellular Physiology Cell Physiol Biochem 2018;47:694-706

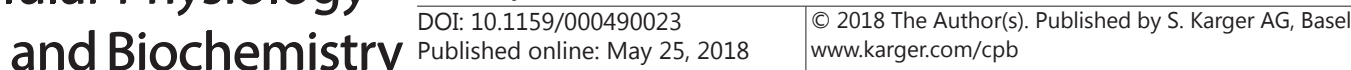 \\ Huang et al.: Icariin Protects Against Osteonecrosis}

\section{ICA suppressed adipogenic differentiation of BMSCs treated with MPS}

As shown in Fig. 3A, after treatment of BMSCs with MPS or MPS + ICA $\left(10^{-5} \mathrm{M}-10^{-7} \mathrm{M}\right)$ for 14 days, the TG content was significantly higher in the MPS-treated group than the control group $(P<0.05)$, and a lower content of TG was observed in the MPS + ICA group compared to the MPS group $(P<0.05)$, especially in the MPS $+\mathrm{ICA}^{-6} \mathrm{M}$ group.

Oil red 0 staining showed the presence of more fat droplets in the MPS group compared with the control group, while the number and size of fat droplets were both significantly decreased after treatment with MPS and $10^{-6}$ M ICA compared to the MPS alone group (Fig. 3B).

Our results indicated that the mRNA level of PPAR $\gamma$ was significantly up-regulated by MPS $(P<0.05)$, as shown in Fig. 3C, while expression in the MPS $+\mathrm{ICA}^{-6} \mathrm{M}$ group was decreased compared to that in the MPS group $(P<0.05)$. Neither MPS nor ICA affected the mRNA expression of P-gp in adipogenic differentiation of BMSCs $(P>0.05)$. As shown in the western blot analysis (Fig. 3D), the expression of PPAR $\gamma$ was up-regulated by MPS, while expression in the MPS + ICA ${ }^{-6} \mathrm{M}$ group was lower than in the MPS group. In contrast P-gp expression showed no difference among the three groups. Immunofluorescence staining revealed that the expression of PPAR $\gamma$ in the MPS group was higher than in the control group, while compared with the MPS group, the MPS $+\mathrm{ICA}^{-6} \mathrm{M}$ group showed a decrease in the expression of PPAR $\gamma$ (Fig. 4B).

\section{ICA increased serum ALP and decreased serum TG in rats treated with MPS}

Serum levels of ALP and TG were measured after 12 weeks' intervention. As shown in Fig. 5A, the ALP level in the MPS group was lower than in the control group, but the difference was not significant $(P>0.05)$. However, we detected a significantly higher ALP level in the rats treated with MPS and ICA $(P<0.05)$. As shown in Fig. 5B, the MPS group had higher serum levels of TG compared to the control group $(P<0.05)$, while the addition of ICA clearly decreased its production.

Fig. 4. Expression of Runx2 in BMSCs cultured in osteogenic medium and of PPAR $\gamma$ in BMSCs cultured in adipogenic medium detected by immunofluorescence; red or green staining shows expressed proteins and blue staining shows the nucleus. (A) Expression of Runx2 was

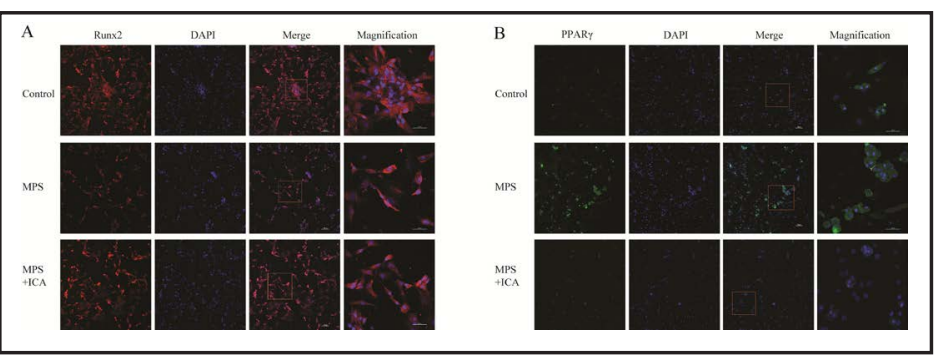
decreased by MPS, while MPS + ICA clearly improved Runx2 expression. (B) Immunofluorescence staining of PPAR $\gamma$ showed the opposite results to Runx2.

Fig. 5. Analysis of serum ALP (A) and TG (B) in SD rats. Values were expressed as the mean absorbance value $(\%) \pm \operatorname{SD}(\mathrm{n}=10) .{ }^{*} \mathrm{P}<0.05$ vs. MPS group.

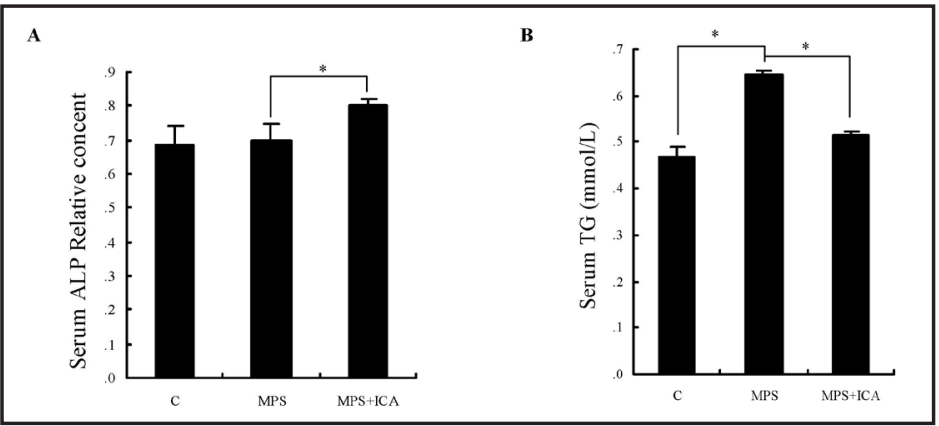




\section{Cellular Physiology Cell Physiol Biochem 2018;47:694-706 \begin{tabular}{l|l|l} 
DOI: 10.1159/000490023 & $\begin{array}{l}\text { O } 2018 \text { The Author(s). Published by S. Karger AG, Basel } \\
\text { www.karger.com/cpb }\end{array}$ \\
\hline and Biochemistry
\end{tabular}

\section{ICA prevents bone loss in a rat model of GC-related ONFH}

We used micro-CT to analyze trabecular changes in the subchondral area of the femoral heads. After 12 weeks, the incidence of osteonecrosis was $0(0 / 10), 70 \%(7 / 20)$ and $30 \%$ $(3 / 10)$ in the control group, MPS group and MPS + ICA group respectively $(P<0.05)$. Fig. 6A shows representative micro CT images of osteonecrosis. The BMD of the rats in the MPS group was $657.5 \pm 39.4 \mathrm{mg} / \mathrm{cm}^{3}$, which was significantly lower than the control group (861.0 $\left.\pm 27.0 \mathrm{mg} / \mathrm{cm}^{3}\right)$, while supplementation with ICA significantly restored the BMD $(763.7 \pm$ $20.8 \mathrm{mg} / \mathrm{cm}^{3}$ ) (Fig. 6B). Meanwhile, quantitative analysis of the trabeculae showed that other bone parameters such as bone volume/total volume (BV/TV), bone surface/total volume (BS/TV), trabecular number (Tb. N), trabecular thickness (Tb. Th) and trabecular spacing (Tb. Sp) were similar among the three groups (Fig. 6C-G). In addition, H\&E and oil red $\mathrm{O}$ staining showed fat tissue invasion and obvious subchondral necrosis in the subchondral bone trabeculae in the MPS group. In contrast, no such changes were observed in the MPS + ICA group in which there were few smaller fat cells and empty lacunae (Fig. 7A, 7B, 7E). Consistent with $\mathrm{H} \& \mathrm{E}$ and oil red $\mathrm{O}$ staining observations, immunohistochemical staining showed more positive staining of Runx2 expression and less expression of PPAR $\gamma$ in the MPS + ICA group compared with the MPS group (Fig. 7C, 7D, 7F, 7G).

\section{Discussion}

Recently, there has been strong interest in studying the efficacy of ICA as a potential alternative therapy for improving bone formation, preventing bone loss and treating osteoporosis [26, 27]. However, there are few studies focusing on ONFH. To our knowledge, this is the first study to demonstrate that ICA can be an effective agent for prevention of ONFH. In this study, we showed that ICA improved cell proliferation, enhanced osteogenic differentiation and suppressed adipogenic differentiation of MPStreated BMSCs. Moreover, our in vitro study demonstrated that ICA increased the expression of osteogenic-associated genes of BMSCs which were inhibited by MPS and decreased the expression of adipogenic-associated genes of BMSCs stimulated by MPS. In addition, we observed that treatment with ICA clearly prevented

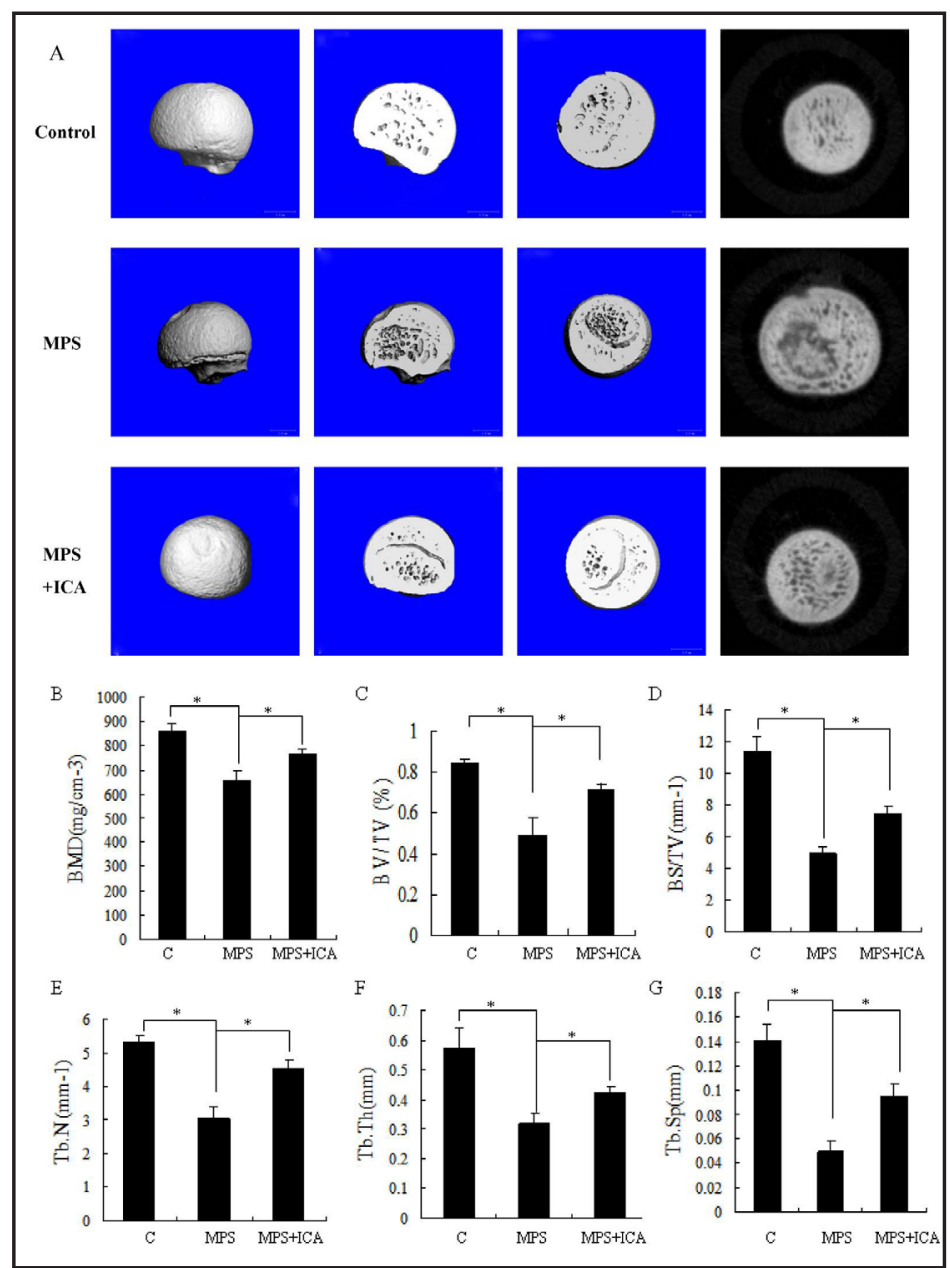

Fig. 6. Micro CT evaluation of the subchondral region of the femoral heads. (A) Two-dimensional images of a coronal section of the femoral heads. (B-G) Morphometric analysis showed callus parameters of the upper outer subchondral bone of the femoral heads. * $\mathrm{P}<0.05$ vs. MPS group. 
Fig. 7. Histological analysis of paraffin sections of the femoral heads. (A) H\&E staining of representative femoral heads in each group. The triangle indicates a broken trabecula and the arrows indicate empty lacunae and hypertrophic fat cells. Sections were stained with oil red $O$ to calculate the percentage of adipocytes in areas (B, E). Immunohistochemical staining of Runx2 and PPAR $\gamma$ in coronal sections of representative femoral heads in each group (C, D, F, G). The arrow indicates the target protein staining. ${ }^{*} \mathrm{P}<0.05$ vs. MPS group.

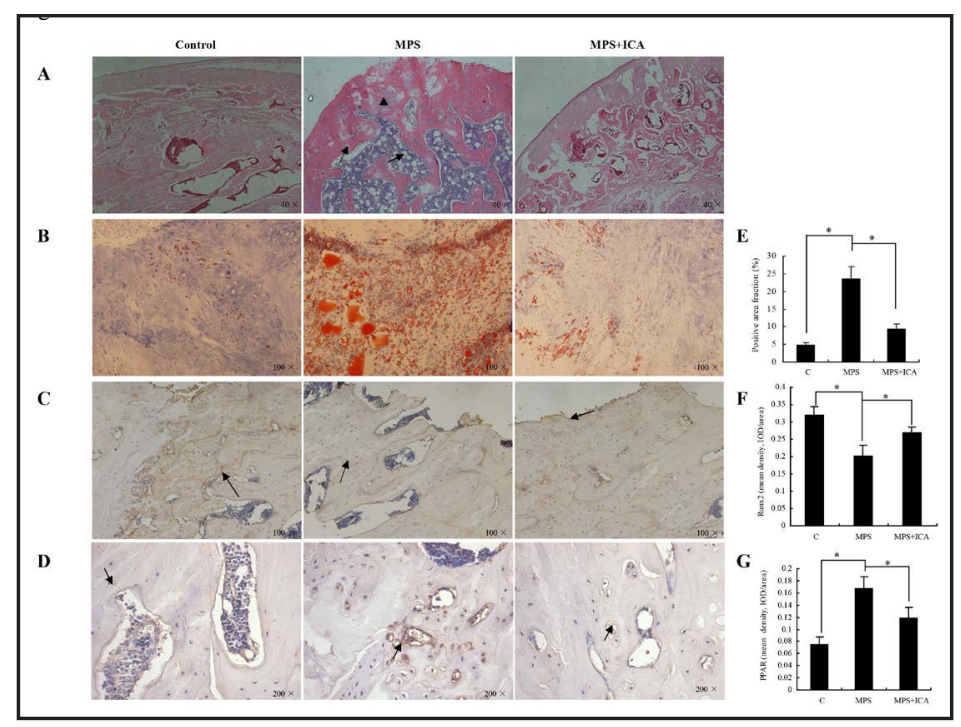

bone loss and reduced the incidence of ONFH at 12 weeks in a rat model of GC-associated ONFH.

Our study demonstrated that treatment of BMSCs with ICA $\left(10^{-9} \mathrm{M}-10^{-5} \mathrm{M}\right)$ significantly increased cell proliferation, especially at $10^{-6} \mathrm{M}$. Many studies indicated that ICA at different concentrations enhanced cell proliferation. Mok et al. found that ICA significantly enhanced proliferation at concentrations ranging from $10^{-14} \mathrm{M}$ to $10^{-6} \mathrm{M}$ in UMR 106 cells [26]. Moreover, a dose-dependent study by Ma revealed that the optimal concentration of ICA for stimulating osteogenesis was $10^{-5} \mathrm{M}$ in ROB cells [28]. These differences in the effective concentration of ICA were due to the differences in the cells used. However, few studies have reported the effect of ICA on BMSCs. Moreover, we also discovered that ICA clearly increased proliferation of BMSCs inhibited by MPS. As BMSCs are essential for the healing response [23, 27] and decreasing activity of BMSCs after exposure to high-dose steroids might be a triggering step of GC-associated ONFH [29], our results on BMSC proliferation with ICA treatment might suggest a potential remedy for the insufficient repair mechanism in GC-associated ONFH.

In the MPS groups, the actual GC dose is that of dexamethasone $\left(10^{-7} \mathrm{M}\right)+\mathrm{MPS}$. However, under physiological conditions, serum cortisol concentrations reach $10^{-8}-10^{-7} \mathrm{M}$ in humans

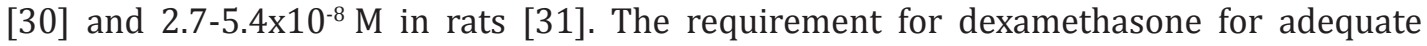
osteogenesis and adipogenesis in in vitro experiments has been reported previously [32,33]. Dexamethasone regulates gene expression in differentiating cells and induces the affinity of the GC receptor for its target sequence in the genome [34]. Moreover, the medium in all groups shared the same concentration of dexamethasone, and the influence of MPS on osteogenesis of BMSCs treated with dexamethasone has been previously reported [35]. In addition, the dose of MPS was 500 times greater than that of dexamethasone in the present study. The effects of ICA on osteogenesis and adipogenesis following treatment with MPS would not be affected by dexamethasone.

BMSCs are multipotent cells that can differentiate into multiple cell lineages. Osteogenic and adipogenic differentiation of BMSCs maintains dynamic balance in the physiological state, but this balance may be disrupted by steroids. Long-term use of high doses of GC can directly destroy the balance by downregulating osteogenic-related genes and upregulating the expression of adipogenic-associated genes [7]. This is considered to be a principal mechanism leading to GC-related ONFH. Accordingly, how to inhibit adipogenic differentiation of BMSCs and promote their osteogenic differentiation is a key issue in the management of ONFH progression.

Our study indicated that MPS clearly induced the differentiation of BMSCs into adipocytes and inhibited their differentiation into osteoblasts. Although the direct effects of ICA in modulating osteogenic differentiation and inhibiting adipogenic differentiation have 
been reported [36, 37], its effects on BMSCs exposed to MPS and treated with ICA have not been studied. Our study further demonstrated that ICA promoted osteogenic differentiation and suppressed adipogenic differentiation of BMSCs treated with MPS.

We found that ICA promoted the osteogenic effect of BMSCs inhibited by MPS and enhanced the biomechanical strength of the femurs in a rat model of GC-associated ONFH. A previous study has reported that osteoblasts differentiated from BMSCs can facilitate new bone formation [38]. However, there is also the possibility that BMSCs might differentiate into the adipocyte lineage when exposed to steroids, and then inhibit osteogenic differentiation, thus resulting in inadequate bone repair in GC-induced ONFH [39]. These findings are consistent with our experimental results, in which we found that lesions in rats of the MPS group showed empty lacunae accompanied by surrounding marrow cell necrosis and occupation of adipocytes during histopathological examination. After ICA treatment, the ratio of empty lacunae and the area of bone marrow occupied by adipocytes were significantly reduced, suggesting that improvement in local adipogenesis was induced by ICA. In addition, ICA increased bone formation and improved the trabecular microarchitecture in the femoral head. These findings suggested that ICA could prevent bone loss induced by MPS.

There are many signaling pathways involved in promoting osteogenic and/or adipogenic lineage differentiation of BMSCs, of which the two most important are the BMP2/Runx2 and the PPAR $\gamma$ signaling pathways [40]. Runx2 is the key transcription factor in osteogenesis and is regulated by BMP-2 [41]. Likewise, PPAR $\gamma$ is the master regulator of adipogenesis and also has been well described to have anti-osteoblastogenic effects. Together, they regulate various cytokines to determine the choice of osteogenic versus adipogenic BMSCs differentiation. Typically, stimulating one pathway is associated with suppression of the other [42, 43]. Although studies have shown that ICA produces higher ALP activity and expression of Runx2, OC and BMP2 levels in vitro [27, 28]; the combined effect of ICA and MPS on BMSCs has rarely been reported. In addition, PPAR $\gamma$ is well known to be upregulated as an adipogenic marker in GC-induced ONFH [44], but few studies have reported the effect of ICA on adipogenic differentiation of BMSCs induced by GC. The present study first demonstrated that ICA regulated both osteogenic and adipogenic differentiation of BMSCs treated with MPS in both in vitro and in vivo experiments. The ICA + MPS group showed higher expression of Runx2 and BMP2, and lower PPAR $\gamma$ expression than the MPS group, suggesting that ICA might mediate promotion of osteogenesis in the BMP2/Runx2 signaling pathway and inhibit adipogenesis through the PPAR $\gamma$-mediated pathway in GC-induced ONFH.

The mechanism via which ICA regulates the pathways of BMP2/Runx2 and PPAR $\gamma$ in GC-induced ONFH is uncertain. Glucocorticoid has a positive relation to oxidative stress production [45] and the presence of DNA oxidation injury was confirmed in the early period of corticosteroid treatment [46]. The important point of oxidative stress modulates osteogenic and adipogenic differentiation has received much attention [47]. Recently, the activity of ICA supplementation in reducing oxidative stress has been reported [48] and confirmed in our previous study [14]. Thus, we speculate that ICA regulates the pathways of BMP2/Runx2 and PPAR $\gamma$ by reducing oxidative stress in GC-induced ONFH. Moreover, estrogen-like activities of ICA have been widely reported [26] and estrogen-like analogues can also reduce oxidative stress [49]. Such a mechanism might be partly involved in regulating oxidative stress through the ER. However, this remains to be studied further.

GC induced osteoblast apoptosis was regarded as the common mechanism in the progress of osteoporosis and osteonecrosis. However, balance disruption between osteogenic and adipogenic differentiation and increased bone marrow fat were also important mechanisms in GC-induced ONFH [7, 50]. In our present study, we not only showed the inducing osteogenic effects of ICA, but also demonstrated its' inhibiting adipogenic effects. Feng R et al. showed that ICA prevented GC induced apoptosis in a GC-induced osteoporosis animal model [51]. The establishment of animal model is different with our animal model of GC-induced ONFH. Moreover, osteoporosis is a systemic disease and ONFH is the disease limited to the femoral head. Osteonecrosis may occur without GC-induced osteoporosis [52]. There are many effective drugs for the treatment of GC-induced osteoporosis such as, 
bisphosphonates, teriparatide, denosumab and etc. The effect is limited when prescribed these drugs in ONFH $[53,54]$. GC-induced ONFH occurs predominantly in younger patients and it is the most common cause of total hip replacement in young adults [50]. Since these replacements have about a 10-year lifespan, any delay in the need for surgery would be welcome [54]. For these reasons, efforts should be made to preserve the joint and delay hip replacement. Because of the potential effect of ICA in GC-induced osteoporosis treatment, it is possible and necessary to research the effect of ICA in GC-induced ONFH. As far as we know, the present study was first to evaluate the effect of ICA in GC-induced ONFH. We will establish the GC-induced ONFH model of core decompression + ICA sustained-release for further understanding of this compound in GC-induced ONFH. If confirmed, ICA will be a promising drug for the treatment and prevention of early stage of GC-induced ONFH.

\section{Conclusion}

In conclusion, the results of our study provided, for the first time, evidence that ICA is an effective compound for promoting bone repair and preventing or delaying the progression of GC-associated ONFH by regulating the BMP2/Runx2 and PPAR $\gamma$-mediated signaling pathways. Thus, ICA should be considered as a potential candidate for management of GCassociated ONFH.

\section{Acknowledgements}

This work was supported by the Natural Science Foundation of Hubei Province (2013CFB376, 2018CFB095). The funders had no role in study design, data collection and analysis, decision to publish, or preparation of the manuscript.

\section{Disclosure Statement}

The authors declare that they have no competing interests.

\section{References}

1 Assouline-Dayan Y, Chang C, Greenspan A, Shoenfeld Y, Gershwin ME: Pathogenesis and natural history of osteonecrosis. Semin Arthritis Rheum 2002;32:94-124.

2 Chan MH, Chan PK, Griffith JF, Chan IH, Lit LC, Wong CK, Antonio GE, Liu EY, Hui DS, Suen MW, Ahuja AT, Sung JJ, Lam CW: Steroid-induced osteonecrosis in severe acute respiratory syndrome: a retrospective analysis of biochemical markers of bone metabolism and corticosteroid therapy. Pathology 2006;38:229235.

3 Duran-Barragan S, Sandoval-Garcia L, Navarrete-Lorenzon M: Additive risk factors resulting in severe osteonecrosis in systemic lupus erythematosus. Arthritis Rheum 2010;62:3276.

-4 Kaste SC, Pei D, Cheng C, Neel MD, Bowman WP, Ribeiro RC, Metzger ML, Bhojwani D, Inaba H, Campbell P, Rubnitz JE, Jeha S, Sandlund JT, Downing JR, Relling MV, Pui CH, Howard SC: Utility of early screening magnetic resonance imaging for extensive hip osteonecrosis in pediatric patients treated with glucocorticoids. J Clin Oncol 2015;33:610-615.

-5 Marston SB, Gillingham K, Bailey RF, Cheng EY: Osteonecrosis of the femoral head after solid organ transplantation: a prospective study. J Bone Joint Surg Am 2002;84-A:2145-2151.

6 Talamo G, Angtuaco E, Walker RC, Dong L, Miceli MH, Zangari M, Tricot G, Barlogie B, Anaissie E: Avascular necrosis of femoral and/or humeral heads in multiple myeloma: results of a prospective study of patients treated with dexamethasone-based regimens and high-dose chemotherapy. J Clin Oncol 2005;23:52175223.

7 Li X, Jin L, Cui Q, Wang GJ, Balian G: Steroid effects on osteogenesis through mesenchymal cell gene expression. Osteoporos Int 2005;16:101-108.

-8 Murata M, Kumagai K, Miyata N, Osaki M, Shindo H: Osteonecrosis in stroke-prone spontaneously hypertensive rats: effect of glucocorticoid. J Orthop Sci 2007;12:289-295.

-9 Urbaniak JR, Seaber AV, Chen LE: Assessment of ischemia and reperfusion injury. Clin Orthop Relat Res 1997;30-36. 


\section{Cellular Physiology Cell Physiol Biochem 2018;47:694-706 $\begin{array}{lll}\text { DOI: 10.1159/000490023 } & \text { Ond Biochemistry } 2018 \text { The Author(s). Published by S. Karger AG, Basel } \\ \text { wwww.karger.com/cpb }\end{array}$}

Huang et al.: Icariin Protects Against Osteonecrosis

10 Chen C, Yang S, Feng Y, Wu X, Chen D, Yu Q, Wang X, Li J, Chen J: Impairment of two types of circulating endothelial progenitor cells in patients with glucocorticoid-induced avascular osteonecrosis of the femoral head. Joint Bone Spine 2013;80:70-76.

$\checkmark 11$ Yeh CH, Chang JK, Wang YH, Ho ML, Wang GJ: Ethanol may suppress Wnt/beta-catenin signaling on human bone marrow stroma cells: a preliminary study. Clin Orthop Relat Res 2008;466:1047-1053.

12 Cuadrado MJ, Lopez-Pedrera C: Antiphospholipid syndrome. Clin Exp Med 2003;3:129-139.

-13 Drescher W, Varoga D, Liebs TR, Lohse J, Herdegen T, Hassenpflug J, Pufe T: Femoral artery constriction by norepinephrine is enhanced by methylprednisolone in a rat model. J Bone Joint Surg Am 2006;88 Suppl 3:162-166.

14 Sun ZB, Wang JW, Xiao H, Zhang QS, Kan WS, Mo FB, Hu S, Ye SN: Icariin may benefit the mesenchymal stem cells of patients with steroid-associated osteonecrosis by ABCB1-promoter demethylation: a preliminary study. Osteoporos Int 2015;26:187-197.

15 Shen P, Wong SP, Yong EL: Sensitive and rapid method to quantify icaritin and desmethylicaritin in human serum using gas chromatography-mass spectrometry. J Chromatogr B Analyt Technol Biomed Life Sci 2007;857:47-52.

16 Griffith JF, Antonio GE, Kumta SM, Hui DS, Wong JK, Joynt GM, Wu AK, Cheung AY, Chiu KH, Chan KM, Leung PC, Ahuja AT: Osteonecrosis of hip and knee in patients with severe acute respiratory syndrome treated with steroids. Radiology 2005;235:168-175.

-17 Li ZR, Sun W, Qu H, Zhou YX, Dou BX, Shi ZC, Zhang NF, Cheng XG, Wang DL, Guo WS: [Clinical research of correlation between osteonecrosis and steroid]. Zhonghua Wai Ke Za Zhi 2005;43:1048-1053.

18 Lau TF, Leung PC, Wong EL, Fong C, Cheng KF, Zhang SC, Lam CW, Wong V, Choy KM, Ko WM: Using herbal medicine as a means of prevention experience during the SARS crisis. Am J Chin Med 2005;33:345-356.

19 Liu Y, Huang L, Hao B, Li H, Zhu S, Wang Q, Li R, Xu Y, Zhang X: Use of an Osteoblast Overload Damage Model to Probe the Effect of Icariin on the Proliferation, Differentiation and Mineralization of MC3T3-E1 Cells through the Wnt/beta-Catenin Signalling Pathway. Cell Physiol Biochem 2017;41:1605-1615.

20 Nian H, Ma MH, Nian SS, Xu LL: Antiosteoporotic activity of icariin in ovariectomized rats. Phytomedicine 2009;16:320-326.

21 Li GW, Xu Z, Chang SX, Nian H, Wang XY, Qin LD: Icariin prevents ovariectomy-induced bone loss and lowers marrow adipogenesis. Menopause 2014;21:1007-1016.

22 Neve A, Corrado A, Cantatore FP: Osteocalcin: skeletal and extra-skeletal effects. J Cell Physiol 2013;228:1149-1153.

23 Kerachian MA, Seguin C, Harvey EJ: Glucocorticoids in osteonecrosis of the femoral head: a new understanding of the mechanisms of action. J Steroid Biochem Mol Biol 2009;114:121-128.

24 Dong Y, Li Y, Huang C, Gao K, Weng X: Systemic application of teriparatide for steroid induced osteonecrosis in a rat model. BMC Musculoskelet Disord 2015;16:163.

-25 Irisa T, Yamamoto T, Miyanishi K, Yamashita A, Iwamoto Y, Sugioka Y, Sueishi K: Osteonecrosis induced by a single administration of low-dose lipopolysaccharide in rabbits. Bone 2001;28:641-649.

-26 Mok SK, Chen WF, Lai WP, Leung PC, Wang XL, Yao XS, Wong MS: Icariin protects against bone loss induced by oestrogen deficiency and activates oestrogen receptor-dependent osteoblastic functions in UMR 106 cells. Br J Pharmacol 2010;159:939-949.

27 Ming LG, Chen KM, Xian CJ: Functions and action mechanisms of flavonoids genistein and icariin in regulating bone remodeling. J Cell Physiol 2013;228:513-521.

28 Ma HP, Ming LG, Ge BF, Zhai YK, Song P, Xian CJ, Chen KM: Icariin is more potent than genistein in promoting osteoblast differentiation and mineralization in vitro. J Cell Biochem 2011;112:916-923.

29 Hernigou P, Beaujean F, Lambotte JC: Decrease in the mesenchymal stem-cell pool in the proximal femur in corticosteroid-induced osteonecrosis. J Bone Joint Surg Br 1999;81:349-355.

30 Bergendahl M, Vance ML, Iranmanesh A, Thorner MO, Veldhuis JD: Fasting as a metabolic stress paradigm selectively amplifies cortisol secretory burst mass and delays the time of maximal nyctohemeral cortisol concentrations in healthy men. J Clin Endocrinol Metab 1996;81:692-699.

-31 Zhao M, Yu Z, Zhang Y, Huang X, Hou J, Zhao Y, Luo W, Chen L, Ou L, Li H, Zhang J: Iron-induced neuronal damage in a rat model of post-traumatic stress disorder. Neuroscience 2016;330:90-99.

32 Cao Y, Gomes SA, Rangel EB, Paulino EC, Fonseca TL, Li J, Teixeira MB, Gouveia CH, Bianco AC, Kapiloff MS, Balkan W, Hare JM: S-nitrosoglutathione reductase-dependent PPARgamma denitrosylation participates in MSC-derived adipogenesis and osteogenesis. J Clin Invest 2015;125:1679-1691. 


\section{Cellular Physiology Cell Physiol Biochem 2018;47:694-706 \begin{tabular}{ll|l} 
DOI: 10.1159/000490023 & and Biochemistry 2018 The Author(s). Published by S. Karger AG, Basel \\
Published online: May 25, 2018 & $\begin{array}{l}\text { www.karger.com/cpb } \\
\text { and }\end{array}$
\end{tabular}}

Huang et al.: Icariin Protects Against Osteonecrosis

-33 Hanada K, Dennis JE, Caplan AI: Stimulatory effects of basic fibroblast growth factor and bone morphogenetic protein-2 on osteogenic differentiation of rat bone marrow-derived mesenchymal stem cells. J Bone Miner Res 1997;12:1606-1614.

34 Becker PB, Gloss B, Schmid W, Strahle U, Schutz G: In vivo protein-DNA interactions in a glucocorticoid response element require the presence of the hormone. Nature 1986;324:686-688.

-35 Zhang YL, Yin JH, Ding H, Zhang W, Zhang CQ, Gao YS: Vitamin K2 Prevents Glucocorticoid-induced Osteonecrosis of the Femoral Head in Rats. Int J Biol Sci 2016;12:347-358.

-36 Zhao J, Ohba S, Shinkai M, Chung UI, Nagamune T: Icariin induces osteogenic differentiation in vitro in a BMP- and Runx2-dependent manner. Biochem Biophys Res Commun 2008;369:444-448.

37 Zhang D, Fong C, Jia Z, Cui L, Yao X, Yang M: Icariin Stimulates Differentiation and Suppresses Adipocytic Transdifferentiation of Primary Osteoblasts Through Estrogen Receptor-Mediated Pathway. Calcif Tissue Int 2016;99:187-198.

-38 Xie XH, Wang XL, He YX, Liu Z, Sheng H, Zhang G, Qin L: Promotion of bone repair by implantation of cryopreserved bone marrow-derived mononuclear cells in a rabbit model of steroid-associated osteonecrosis. Arthritis Rheum 2012;64:1562-1571.

-39 Sheng HH, Zhang GG, Cheung WH, Chan CW, Wang YX, Lee KM, Wang HF, Leung KS, Qin LL: Elevated adipogenesis of marrow mesenchymal stem cells during early steroid-associated osteonecrosis development. J Orthop Surg Res 2007;2:15.

40 James AW: Review of Signaling Pathways Governing MSC Osteogenic and Adipogenic Differentiation. Scientifica (Cairo) 2013;2013:684736.

41 Peng S, Zhou G, Luk KD, Cheung KM, Li Z, Lam WM, Zhou Z, Lu WW: Strontium promotes osteogenic differentiation of mesenchymal stem cells through the Ras/MAPK signaling pathway. Cell Physiol Biochem 2009;23:165-174.

42 Valenti MT, Garbin U, Pasini A, Zanatta M, Stranieri C, Manfro S, Zucal C, Dalle Carbonare L: Role of ox-PAPCs in the differentiation of mesenchymal stem cells (MSCs) and Runx2 and PPARgamma2 expression in MSCslike of osteoporotic patients. PLoS One 2011;6:e20363.

43 Li X, Cui Q Kao C, Wang GJ, Balian G: Lovastatin inhibits adipogenic and stimulates osteogenic differentiation by suppressing PPARgamma2 and increasing Cbfa1/Runx2 expression in bone marrow mesenchymal cell cultures. Bone 2003;33:652-659.

44 Cui Q Wang GJ, Balian G: Steroid-induced adipogenesis in a pluripotential cell line from bone marrow. J Bone Joint Surg Am 1997;79:1054-1063.

45 Shen C, Cai GQ, Peng JP, Chen XD: Autophagy protects chondrocytes from glucocorticoids-induced apoptosis via ROS/Akt/FOXO3 signaling. Osteoarthritis Cartilage 2015;23:2279-2287.

46 Ichiseki T, Kaneuji A, Katsuda S, Ueda Y, Sugimori T, Matsumoto T: DNA oxidation injury in bone early after steroid administration is involved in the pathogenesis of steroid-induced osteonecrosis. Rheumatology (Oxford) 2005;44:456-460.

47 Atashi F, Modarressi A, Pepper MS: The role of reactive oxygen species in mesenchymal stem cell adipogenic and osteogenic differentiation: a review. Stem Cells Dev 2015;24:1150-1163.

-48 Tang Y, Jacobi A, Vater C, Zou L, Zou X, Stiehler M: Icariin promotes angiogenic differentiation and prevents oxidative stress-induced autophagy in endothelial progenitor cells. Stem Cells 2015;33:1863-1877.

49 Manolagas SC: From estrogen-centric to aging and oxidative stress: a revised perspective of the pathogenesis of osteoporosis. Endocr Rev 2010;31:266-300.

50 Mankin HJ: Nontraumatic necrosis of bone (osteonecrosis). N Engl J Med 1992;326:1473-1479.

-51 Feng R, Feng L, Yuan Z, Wang D, Wang F, Tan B, Han S, Li T, Li D, Han Y: Icariin protects against glucocorticoid-induced osteoporosis in vitro and prevents glucocorticoid-induced osteocyte apoptosis in vivo. Cell Biochem Biophys 2013;67:189-197.

52 Weinstein RS: Clinical practice. Glucocorticoid-induced bone disease. N Engl J Med 2011;365:62-70.

53 Yuan HF, Guo CA, Yan ZQ: The use of bisphosphonate in the treatment of osteonecrosis of the femoral head: a meta-analysis of randomized control trials. Osteoporos Int 2016;27:295-299.

-54 Weinstein RS: Glucocorticoid-induced osteonecrosis. Endocrine 2012;41:183-190. 